1 (MMP3), Collagenase 3 (MMP13), Kallikrein-6 (KLK6), Cathepsin D (CTSD) and Cathepsin E (CTSE) had increased activity whilst Meprin A subunit alpha (MEP1A), Cathepsin B (CTSB) and Granzyme A (GZMA) had reduced activity in HCC compared to controls.

Conclusions Urinary CE-MS analysis identified eight proteases specific to HCC. These proteases could be associated with the development of HCC. Recent cancer research revealed that most of the proteases associated with cancer are involved in the degradation of the extracellular matrix and are also involved in the growth and spreading of cancer in the body.

\section{P175 URINARY ANALYSIS OF HEPATOCELLULAR CARCINOMA PATIENTS USING SOLID PHASE MICROEXTRACTION}

${ }^{1,2}$ Ayman Bannaga*, ${ }^{3}$ Frank Kvasnik, ${ }^{3}$ Krishna Persaud, ${ }^{1,2}$ Ramesh Arasaradnam. ${ }^{1}$ University Hospital Coventry and Warwickshire NHS Trust, Coventry, UK; ${ }^{2}$ Warwick Medical School, University of Warwick, Coventry, UK; ${ }^{3}$ School of Chemical Engineering and Analytical Science, University of Manchester, Coventry, UK

\subsection{6/gutjnl-2020-bsgcampus.250}

Introduction Solid phase microextraction (SPME) is an analytical method for preconcentration of volatile organic compounds (VOCs) commonly used in analysis of biological samples. We applied SPME to study the urinary signatures of VOCs in hepatocellular carcinoma (HCC) patients.

Methods Ethical approval was granted by Coventry and Warwickshire research ethics committee (09/H1211/38). We conducted a prospective recruitment between January to June 2019. Male to female ratio was 5:1 and mean age was 72 years (range 42 to 94). HCC cases were diagnosed as per EASL recommendations for 2018. Controls included patients that were suspected of cancer but had negative investigations. We collected $5 \mathrm{mls}$ of urine from $31 \mathrm{HCC}$ cases and 18 controls. The urine samples were left to freeze within 2 hours to $-80^{\circ} \mathrm{C}$. Analysis of these samples was then completed at the end of study recruitment. Prior to analysis samples were left to thaw in a water bath at $50^{\circ} \mathrm{C}$ for $1 \mathrm{~h}$. Urine was then placed into a Falcon conical centrifuge tube $50-\mathrm{mL}$ with a modified cap with two slots to allow two solid phase microextraction tabs to be inserted to absorb gases from the head space of the samples. The tabs were then analysed using an array of eight metal oxide gas sensors. Responses from the gas sensors (1 to 8 ) to the urinary vapours were captured over a period of 180 seconds. The responses were digitized and stored by a computer software. The method of principal

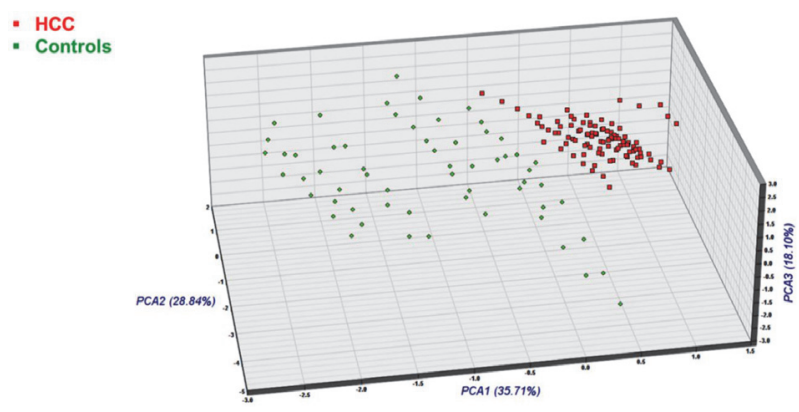

Abstract P175 Figure 1 components analysis (PCA) was then employed to visualize these data.

Results Data from the eight gas sensor responses were then demonstrated on a PCA plot, which made no assumptions about separation between classes. This visually showed that responses from the urinary VOCs of HCC patients were clearly differentiated from controls as shown in the plot below. This gives the impression that HCC has a potential urinary specific chemical signature. Urinary VOCs signature could potentially be used in the diagnosis of HCC.

Conclusions Application of SPME urinary VOCs analysis in HCC patients has future potential as a diagnostic method. This will require further validation from other interested research groups.

\section{P176 TSPAN6: A NOVEL PLAYER IN THE MICROENVIRONMENT OF PRIMARY LIVER CANCERS}

${ }^{1}$ OO Bolimowska*, 'DA Patten, 's Shetty, ${ }^{2} \mathrm{~F}$ Berditchevski, ${ }^{1} \mathrm{JM}$ O'Rourke, ${ }^{10}$ Cain, ${ }^{1} \mathrm{AL}$ Wilkinson. 'Centre for Liver and Gastrointestinal Research, University of Birmingham, UK; ${ }^{2}$ CRUK Institute for Cancer Studies, University of Birmingham, UK

\subsection{6/gutjnl-2020-bsgcampus.251}

Introduction Tetraspanins, a large family of membrane proteins, have been implicated in the regulation of the tumour microenvironment of a number of cancers. TSPAN6 has previously been shown to modulate the immune microenvironment in breast cancers via indirect interactions with tumour-infiltrating B cells; however, TSPAN6 has not been studied within the context of primary liver cancers, nor the human liver in general.

Methods TSPAN6 mRNA expression was quantified in normal, chronically diseased and primary liver cancer tissues. The distribution and cellular localisation of TSPAN6 protein expression was explored utilising immunohistochemistry and multi-colour immunofluorescence. In addition, hepatocellular carcinoma (HCC) tumour samples were histologically scored on intensity and proportion of positivity for TSPAN6 and Kaplan-Meier curves were generated for patients with negative/low expression vs. positive TSPAN6 tumour expression.

Results Transcriptional expression of TSPAN6 was comparable between normal and chronically diseased liver tissues, but was increased in primary liver cancer tissues, compared to matched distal tissues. TSPAN6 was strongly expressed in biliary epithelial cells, and to a lesser degree in hepatocytes within normal tissues and showed increased expression in the diseased state. In chronically diseased tissues, a strong association with the fibrotic septa was observed and we show that TSPAN6 strongly co-localised with cytokeratin 7 , a marker of intermediary cells in the ductular reaction. TSPAN6-expressing cells were also in close association with aggregates of $\mathrm{CD} 20^{+} \mathrm{B}$ cells within diseased tissues. Primary liver cancer tumours showed variable expression of TSPAN6 and preliminary analysis in HCC tumours suggested a correlation between positive tumour TSPAN6 expression and better overall patient survival, over a 5 year period.

Conclusions In this study, we have described, for the first time, the expression of TSPAN6 in human liver tissues. Chronically diseased liver tissues showed increased protein expression of TSPAN6 compared to normal tissues, and its expression was largely associated with the fibrotic septa and 